\title{
A Planning Method of Hybrid AC/DC Receiving End Grid with Mutually Exclusive Constraints
}

\author{
Yi Luo ${ }^{1}$, Yin Zhang ${ }^{1}$, Muyi Tang ${ }^{*}$, Youbin Zhou ${ }^{2}$, Ying Wang ${ }^{2}$, Defu Cai ${ }^{2}$, Haiguang Liu ${ }^{2}$, Ding Li ${ }^{1}$, Yaning Wu ${ }^{1}$ and Yue \\ $\operatorname{Han}^{1}$ \\ ${ }^{1}$ School of Electrical and Electronic Engineering, Huazhong University of Science and Technology, Wuhan, Hubei, 430074, China \\ ${ }^{2}$ Electric Power Research Institute of Hubei Electric Power Company, Wuhan, Hubei, 430074, China
}

\begin{abstract}
With the large-scale application of LCC-HVDC and VSC-HVDC in power systems, the mutually exclusive constraints (MES) appear in the optimal planning of hybrid AC/DC receiving end power grid. The MES increase the scale of the construction set of substations and the transmission lines and decrease the planning efficiency and effectiveness of the conventional method. This paper proposes a novel hybrid AC/DC receiving end grid planning method with MES. Constraint satisfaction problem (CSP) is utilized to model the set of mutually exclusive selected lines, in which mutually exclusive candidate lines are converted to mutually exclusive variables and then introduced into the planning model as constraints. After establishing the hybrid AC/DC receiving end grid planning model with MES, the backtracking search algorithm (BSA) is used to solve the optimal planning. The effectiveness of the proposed hybrid AC/DC power grid planning method with MES is verified by case studies.
\end{abstract}

\section{Introduction}

With the rapid development of VSC-HVDC and LCCHVDC in power systems, the power systems present the complex characteristics of multiple voltage levels combination, $\mathrm{AC} / \mathrm{DC}$ hybrid, multi-level, flexible selfgrouping. The unique architecture of the power systems makes the network planning of the $\mathrm{AC} / \mathrm{DC}$ receiving end power grid more complicated. Conventional planning methods generally search the optimal planning scheme from the known candidate converter stations sets and DC transmission lines sets respectively. Obviously, it is not consistent with the connotation of optimal planning to divide grid planning into two or more independent stages artificially. Besides, in AC/DC receiving end power grid planning, the existence of MES greatly increases the number of construction combinations, and reduces the possibility of obtaining optimal planning through conventional methods. It is necessary to improve the method of receiving end network planning from methodology.

The research on the optimal planning of the hybrid $\mathrm{AC} / \mathrm{DC}$ receiving end gird is still in the initial stage [1]. Reference [2] summarizes the factors that affect the capacity planning of VSC-HVDC converter stations in the $\mathrm{AC} / \mathrm{DC}$ systems and proposes a planning method for hybrid AC/DC systems including VSC-HVDC with large-scale renewable energy integration. Taken optimal power flow into consideration, reference [3] decomposes the optimal AC/DC gird planning into two nested problems, including optimal AC/DC line configuration problem and optimal power flow problem. To handle the uncertainty of RES, a multistage $\mathrm{AC} / \mathrm{DC}$ grid expansion planning model is presented in [4], in which uncertainty is modeled through multistage scenario tree. Reference [5] analyses the difficulties and risks of grid planning under multiple AC/DC lines feed-in, a VSC-HVDC planning model is proposed to maximize transmission section capacity. The above studies have carried out preliminary research on the hybrid $\mathrm{AC} / \mathrm{DC}$ grid planning, but they have not fully considered mutually exclusive constraints, with which the uncertain of line type will lead to the huge candidate sets and the conventional methods are unable to get the optimal results.

Based on this, this paper proposes a hybrid AC/DC receiving end grid planning method considering MES. The CSP in the field of artificial intelligence is applied to modify the general planning model and to deal with MES. Introduce mutually exclusive candidate lines in model constraints by mutually exclusive variables, form individual constraint sets of mutually exclusive lines. Then, the MES are fully embodied in the programming model. The BSA is used to solve the model. Finally, the planning results of $\mathrm{AC} / \mathrm{DC}$ grid planning with MES, respectively got by the proposed planning approach and conventional approach, are compared, and the effectiveness of the proposed method is verified.

\section{The description of MES in CSP}

In this paper, the MES in AC/DC grid planning mainly refers to the mutually exclusive phenomenon of the candidate transmission line set, that is, only one type of

\footnotetext{
"Corresponding author's e-mail: M201971388@hust.edu.cn
} 
AC line, LCC-HVDC line, and VSC-HVDC line can be built between point A and B. Assume the variable $\alpha_{i}$ is the type variable of line i, $\alpha_{i}$ satisfies equation (1):

$$
\alpha_{i}= \begin{cases}1 & \text { AC line } \\ 2 & \text { LCC-HVDC line } \\ 3 & \text { VSC-HVDC line }\end{cases}
$$

Different type corresponds to different operation and construction constraint, as in equation (2):

$$
F\left(\alpha_{i}=x\right) \cap F\left(\alpha_{i}=y\right)=\varnothing \quad x \neq y \quad x \in\{1,2,3\} \quad y \in\{1,2,3\}
$$

where $F\left(\alpha_{i}=x\right)$ represents the model constraints of transmission line $i$.

In the structure of CSP, the mutually exclusive variables set and mutually exclusive variables range set are used to represent the MES in the planning line set. The formations of the mutually exclusive variables set $\mathbf{X}_{1}$ and the mutually exclusive variables range set $\mathbf{D}_{1}$ are shown in equation (3)-(4):

$$
\begin{gathered}
\mathbf{X}_{1}=\left\{\left\{Z_{1}, \alpha_{1}\right\},\left\{Z_{2}, \alpha_{2}\right\}, \ldots,\left\{Z_{i}, \alpha_{i}\right\}, \ldots\right\} \quad i=1,2, \ldots, \mathrm{K} \\
\left\{\begin{array}{r}
\mathbf{D}_{1}=\left\{\left\{d_{z_{1}}, d_{\alpha_{1}}\right\},\left\{d_{z_{2}}, d_{\alpha_{2}}\right\}, \ldots,\left\{d_{z_{i}}, d_{\alpha_{i}}\right\}, \ldots\right\} \\
d_{z_{i}} \in\{0,1\}, \quad d_{\alpha_{i}} \in\{1,2,3\}
\end{array}\right.
\end{gathered}
$$

where $\mathbf{X}_{1}$ is the state variable set of transmission line with MES. $Z_{i}$ is the construction state variable of line $i$ with MES. $\alpha_{i}$ is the construction type of line $i . \mathbf{D}_{1}$ is the range of state variable. $d_{z_{i}}$ is the range of construction state, $Z_{i}=0, Z_{i}=1$ respectively represent line $i$ is not put into construction and put into construction. $d_{\alpha_{i}}$ is the range of construction type, the range of $\alpha_{i}$ and its implication have been shown above.

\section{CSP planning model of AC/DC receiving end grid considering MES}

The CSP description of MES is introduced into the conventional planning model, and the CSP planning model of $\mathrm{AC} / \mathrm{DC}$ receiving end grid considering the MES is obtained.

\subsection{0bjective function}

$$
\begin{gathered}
\min \left[\sum_{i=1}^{N_{\mathrm{L}}}\left(C_{i} Z_{i} L_{i}\right)\right] \cdot \frac{r(1+r)^{\mathrm{LT}}}{(1+r)^{\mathrm{LT}}-1}+F_{\mathrm{M}}+F_{\mathrm{LO}}+F_{\mathrm{RG}}+F_{\mathrm{EP}} \\
F_{\mathrm{M}}=\sum_{i=1}^{N_{\mathrm{L}}} \varepsilon_{i} C_{i} Z_{i} L_{i}
\end{gathered}
$$

$$
\begin{gathered}
F_{\mathrm{LO}}=C_{\mathrm{LOSS}} \cdot E_{\mathrm{LOSS}}, \\
E_{\mathrm{LOSS}}=T_{\max } \cdot \max _{s \in N_{S}}\left\{\sum_{i=1}^{N_{\mathrm{L}-\mathrm{LI}}}\left(Z_{i} \cdot r_{i} \cdot\left(P_{i, t_{0}}^{\mathrm{L}} / U_{n_{i}, t_{0}}\right)^{2}\right)\right\} \\
F_{\mathrm{RG}}=f_{\mathrm{RG}}+f_{\mathrm{RW}}+f_{\mathrm{RH}} \\
f_{\mathrm{RG}}=T_{\mathrm{RG}} \cdot C_{\mathrm{G}-\mathrm{G}} \cdot \sum_{i=1}^{N_{\mathrm{G}}^{-}}\left(P_{i, t_{1}, 0}^{\mathrm{G}}-P_{i, t_{1}}^{\mathrm{G}}\right) \\
\sum_{i=1}^{N_{\mathrm{G}}}\left(P_{i, t_{1}, 0}^{\mathrm{G}}-P_{i, t_{1}}^{\mathrm{G}}\right)=0 \\
f_{\mathrm{RW}}=C_{\mathrm{G}} \cdot T_{\mathrm{RG}} \cdot \sum_{i=1}^{N_{\mathrm{W}}} \Delta P_{i, t_{1}}^{\mathrm{W}}, f_{\mathrm{RH}}=C_{\mathrm{G}} \cdot T_{\mathrm{RG}} \cdot \sum_{i=1}^{N_{\mathrm{H}}} \Delta P_{i, t_{1}}^{\mathrm{H}}
\end{gathered}
$$

Where $r$ is the annual interest rate. LT is the lifetime of the construction line. $N_{\mathrm{L}}$ is the number of candidate lines. $C_{i}$ is the unit investment cost of new lines. $L_{i}$ is the line length. $F_{\mathrm{M}}, F_{\mathrm{LO}}, F_{\mathrm{RG}}$ respectively describe the maintenance costs, network loss cost, network congestion cost. $\varepsilon_{i}$ represents the maintenance cost ratio. $C_{\text {LOSS }}$ represents the network loss cost factor. $E_{\text {LOSS }}$ indicates the total network loss power. $T_{\max }$ is the maximum network loss duration time. $t_{0}$ is the maximum network loss moment. $N_{\text {L-All }}$ is the operation line set. $P_{i, t_{0}}^{\mathrm{L}}$ is the power of line $i$ at maximum network loss moment. $U_{n_{i}, t_{0}}$ is the voltage of the node connected to line $i . r_{i}$ is the resistance of the line. $f_{\mathrm{RG}}, f_{\mathrm{RW}}, f_{\mathrm{RH}}$ respectively describe the congestion cost of the thermal unit, the wind farm, the hydropower station. $N_{\mathrm{G}}, N_{\mathrm{w}}$, $N_{\mathrm{H}}$ are the set of the thermal unit, the wind farm, the hydropower station respectively. $T_{\mathrm{RG}}$ is the maximum congestion duration time. $C_{\mathrm{G}-\mathrm{G}}$ is the internal power transfer cost of the thermal unit. $C_{\mathrm{G}}$ is the power transfer cost of the wind power and hydropower to the thermal unit. $t_{1}$ is the maximum network congestion moment. $P_{i, t_{1}, 0}^{\mathrm{G}}, P_{i, t_{1}}^{\mathrm{G}}$ stand for the planned and actual thermal power when congestion. $\Delta P_{i, t_{1}}^{\mathrm{W}}, \Delta P_{i, t_{1}}^{\mathrm{H}}$ are the power transfer of wind power and hydropower. Equation (6) is the formula of maintenance cost. Equation (7) introduces maximum network loss to calculate the network loss cost. Equation (8)-(11) calculate the network congestion cost by power transfer cost.

The constraints of the CSP planning problem contain three parts: $\mathbf{X}$, the variable set of candidate lines; $\mathbf{D}$, the range set of candidate line variables; $\mathbf{C}$, the overall constraints of the $\mathrm{AC} / \mathrm{DC}$ grid planning.

\subsection{Variable set $X$ and range set of variable $D$}

$\mathbf{X}=\left\{\left\{Z_{1}, \alpha_{1}\right\},\left\{Z_{2}, \alpha_{2}\right\}, \ldots,\left\{Z_{i}, \alpha_{i}\right\}, \ldots,\left\{Z_{\mathrm{K}}, \alpha_{k}\right\}, \ldots,\left\{Z_{\mathbb{N}}, \alpha_{N}\right\}\right\} \quad i=1,2, \ldots, \mathrm{N}$ 


$$
\left\{\begin{array}{c}
\mathbf{D}=\left\{\left\{d_{z_{1}}, d_{\alpha_{1}}\right\},\left\{d_{z_{2}}, d_{\alpha_{2}}\right\}, \ldots,\left\{d_{z_{i}}, d_{\alpha_{i}}\right\}, \ldots,\left\{d_{z_{\mathrm{K}}}, d_{\alpha_{\mathrm{K}}}\right\}, \ldots,\left\{d_{z_{\mathrm{N}}}, d_{\alpha_{\mathrm{N}}}\right\}\right\} \\
d_{z_{i}} \in\{0,1\}, d_{\alpha_{i}} \in\{1,2,3\}, i=1,2, \ldots, \mathrm{N}
\end{array}\right.
$$

In set $\mathbf{X}$, the first $\mathrm{K}$ candidate lines are lines with MES, the same as equation (3). The other $\mathrm{N}-\mathrm{K}$ candidate lines do not have MES, and for each $i, \alpha_{i}$ is certain. Similar to $\mathbf{X}$, the first $\mathrm{K}$ subsets in set $\mathbf{D}$ are range sets of candidate lines' state variable with MES.

\subsection{AC/ DC grid planning constraints C}

\subsubsection{General constraints $\mathbf{C}_{1}$.}

These are constraints must be followed for grid planning and optimization, and have nothing to do with the type of construction line. Mainly include the line construction time constraint (14), the line construction sequence constraint (15), the power balance constraint (16), the generation output constraint (17), the load supply constraint (18):

$$
\begin{gathered}
Z_{i}= \begin{cases}0 & t<t_{i, \min } \\
1 & t \geq t_{i, \text { max }}\end{cases} \\
Z_{i} \geq Z_{j} \quad \forall t \\
\sum_{i, g}^{\alpha_{n}} P_{i}^{g}-P_{n}^{\mathrm{D}}+P_{n}^{\mathrm{D}, \text { curtail }}=\sum_{i}^{\varphi_{n}} P_{i}^{\mathrm{L}, \text { in }}-\sum_{i}^{\rho_{n}} P_{i}^{\mathrm{L}, \text { out }} \\
P_{i}^{\mathrm{g}, \text { min }} \leq P_{i}^{g} \leq P_{i}^{\mathrm{g}, \max } \\
P_{n}^{\mathrm{D}, \text { curtail }}=0
\end{gathered}
$$

where $t_{i, \min }, t_{i, \max }$ respectively represent the earliest and latest construction time of line $i . \alpha_{n}$ represents the generator set connected to node $n . \varphi_{n}, \rho_{n}$ represent the line sets flow in and out node $n . g$ is the generator type index which includes thermal power, wind power, and hydropower. $P_{i}^{g}$ is the output power of generator $i$ of type $g . P_{i}^{\mathrm{L} \text {,in }}, P_{i}^{\mathrm{L}, \text { out }}$ respectively represent the power of lines flowing in and out node $n . P_{n}^{\mathrm{D}}, P_{n}^{\mathrm{D} \text {,curtail }}$ respectively represent the load power and load curtailment of node $n . P_{i}^{\mathrm{g}, \min }, P_{i}^{\mathrm{g} \text {,max }}$ stand for the minimum output and capacity of generator respectively.

Constraint (14) indicates that according to the construction of power plants and substations, certain lines have restrictions on commissioning stages. Constraint (18) restricts that it is not allowed to curtail load when the system operates normally and N-1 contingency happens.

\subsubsection{Lines' MES constraints $\mathbf{C}_{2}$.}

$\mathbf{C}_{2}$ are related to the construction type of line. In addition to the power flow constraints, VSC-HVDC and LCC-
HVDC lines also have constraints such as converter station capacity constraint.

- $\quad$ MES of AC lines, include the formula of branch power (19), the line's capacity constraint (20):

$$
\begin{gathered}
P_{l}^{\mathrm{L}}=B_{l} \mathbf{A}_{l} \boldsymbol{\theta} \\
-P_{l}^{\mathrm{L}, \max } \leq P_{l}^{\mathrm{L}} \leq P_{l}^{\mathrm{L}, \max }
\end{gathered}
$$

where $P_{l}^{\mathrm{L}}, P_{l}^{\mathrm{L} . \max }$ are respectively the power flow and capacity of line $l . B_{l}$ is the susceptance of line. $\mathbf{A}_{l}$ is the adjacency vector of line $l$ with bus. $\boldsymbol{\theta}$ represents the vector of the phase angle.

- MES of LCC-HVDC lines, include the DC line's capacity constraint (21), the converter stations capacity constraint (22), the multi-infeed short circuit ratio (MSCR) constraint (23):

$$
\begin{gathered}
P_{i}^{\mathrm{DC}}=0 \bigcup P_{i}^{\mathrm{DC}, \text { min }} \leq\left|P_{i}^{\mathrm{DC}}\right| \leq P_{i}^{\mathrm{DC}, \text { max }} \\
S_{i} \leq S_{i}^{\max } \\
\mathrm{MSCR}_{i}=\frac{S_{i}^{\mathrm{Con}}}{P_{i}^{\mathrm{DC}, \max }+\sum_{j=1, j \neq i}^{n} F_{j-i} P_{j}^{\mathrm{DC}, \max }} \geq 3
\end{gathered}
$$

where $P_{i}^{\mathrm{DC}}$ is the transmission power of the DC line. $P_{i}^{\mathrm{DC}, \min }, P_{i}^{\mathrm{DC}, \max }$ represent the minimum and maximum power of the DC line. $S_{i}, S_{i}^{\max }$ are the power and capacity of converter station respectively. $\mathrm{MSCR}_{i}$ is the MSCR of DC line $i . S_{i}^{\text {Con }}$ is the short circuit capacity of converter station bus. $F_{j-i}$ is the impact factor between line $i$ and $j$. Equation (23) indicates that $\mathrm{MSCR}_{i}$ should not be less than 3.

- MES of VSC-HVDC lines, include the power flow direction constraint (24):

$$
\left|P_{t^{\prime}}^{\mathrm{DC}}+P_{t^{\prime}+i}^{\mathrm{DC}}\right|=\left|P_{t^{\prime}}^{\mathrm{DC}}\right|+\left|P_{t^{\prime}+i}^{\mathrm{DC}}\right|, \quad 0<i \leq \mathrm{T}
$$

where $t^{\prime}$ represents the time the power flow direction of DC line change. $T$ represents the minimum time interval of direction change. Constraint (24) means the frequent change of power flow direction is not allowed in operation, so the time interval of direction change of DC line should be limited.

\section{Solution Technique}

The backtracking search algorithm (BSA) with independent pattern coding can solve the subtree CSP [68]. The characteristic of the BSA lies in using the deep search first algorithm to search the subtree, and its biggest advantage is that the subtree can use a constraint set to prune the constraint of a subtree without a solution. Additionally, reference [9] completely proves the characteristics of mutually exclusive resource allocation and independence of the MES. Based on this, this chapter establishes the independent pattern coding BSA 
for mutually exclusive resource allocation, which is used to quickly construct solution space for the resource allocation of the constraint and reduces the size of the solution space. Therefore, this BSA is a potential method to solve the CSP planning model with MES. The detailed process of the power grid planning using BSA is shown in figure 1 .

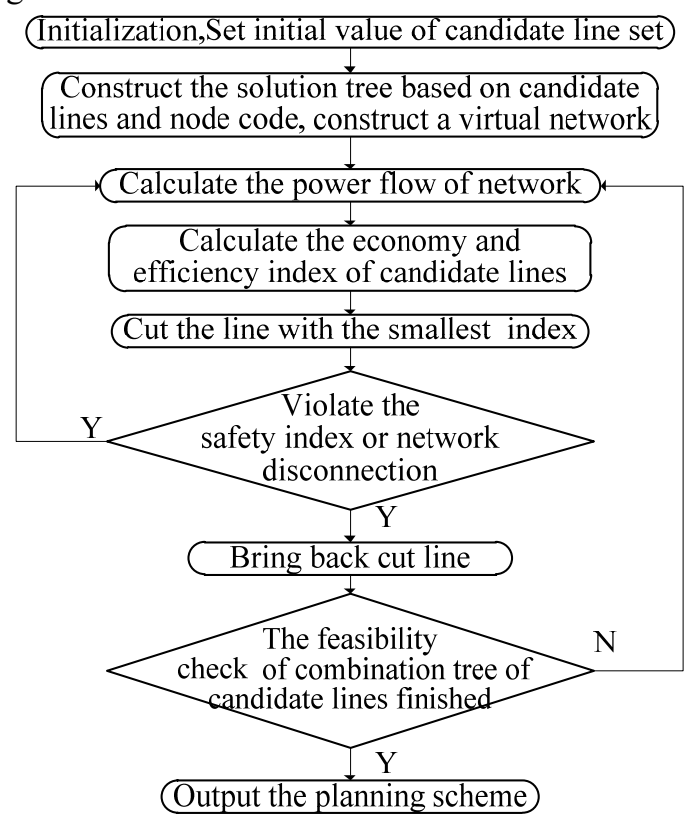

Figure 1. The process of the CSP power grid planning using BSA

\section{Case study}

The case study uses the 18-node system in reference [10] and adds the DC line. The system has 10 nodes and 9 lines, including 2 DC lines. In a future level year, the system will expand to 18 nodes, a total of 33 expandable lines, including LCC-HVDC lines and VSC-HVDC lines. Among the candidate lines, the lines from node 11 to 12 , the lines from node 9 to 10 , the lines from node 7 to 9 have MES, and AC lines, LCC-HVDC or VSC-HVDC lines can be expanded. The initial state of the system is shown in figure 2 , where the solid lines stand for the existing lines and the dotted lines represent the candidate lines.

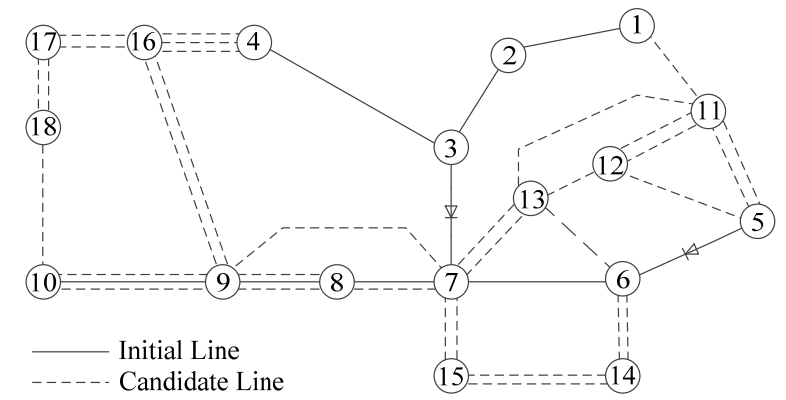

Figure 2. Diagram of case study

The planning scheme obtained by the CSP planning method in this paper is shown in figure 3 , and it is called Plan 1. The initial lines are represented by solid lines, while the selected lines in the planning scheme are marked by dotted lines. In the scheme, a total of 17 new lines will be built, including a VSC-HVDC line from node 11 to 12 and an LCC-HVDC line from node 9 to 10 , with a total length of about $1830 \mathrm{~km}$.

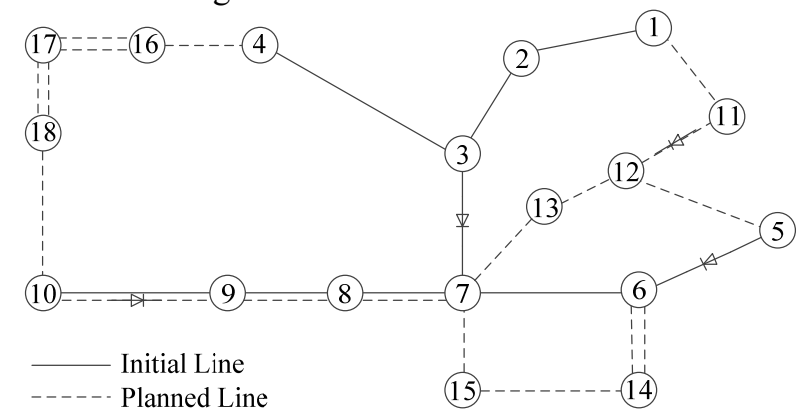

Figure 3. The planning results of the CSP planning model, Plan

The traditional stepwise expansion method is used to calculate the 18-node system expanding problem, and the planning scheme obtained, Plan 2, is shown in figure 4 . The scheme will construct 18 new lines, with a total length of $2005 \mathrm{~km}$.

The detailed parameter comparison between Plan 1 and Plan 2 is listed in table 1. As shown in table 1, figure 3 and figure 4 , the construction cost of Plan 1 is lower and the network structure on the right side of the system is more robust. Additionally, the MSCR of the DC feedout point is greater than that of Plan 2, which is more conducive to the safe operation of power systems. In summary, the proposed method is effective in dealing with $\mathrm{AC} / \mathrm{DC}$ receiving end grid planning with MES and is superior to the traditional method in terms of planning results.

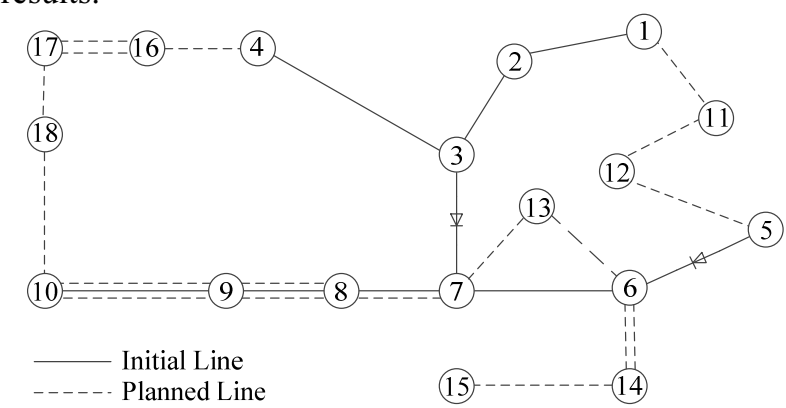

Figure 4. The planning results of the conventional method, Plan 2 
Table 1. Comparison between Plan 1 and Plan 2

\begin{tabular}{lccc}
\hline Scheme & Total number of new lines & Investment cost (CNY) & \multicolumn{2}{c}{ MSCR } \\
\hline Plan 1 & 17 & 214500 & 3.86 \\
Plan2 & 18 & 225800 & 3.123 .733 .583 .11 \\
\hline
\end{tabular}

\section{Conclusion}

In this paper, an optimal planning method for $\mathrm{AC} / \mathrm{DC}$ receiving end grid with MES for the candidate lines is proposed. The CSP theory is used to describe the MES for the candidate lines, and a CSP planning model for the receiving end grid is established. The backtracking search algorithm is used to solve the programming model. The effectiveness of the proposed model for $\mathrm{AC} / \mathrm{DC}$ network planning with mutually exclusive constraints is verified by case studies. In essence, the proposed receiving end grid planning model integrates the step determining DC lines, which is separated from the main planning process in conventional planning method, into the optimization process of network planning, and it avoids human interference when determining the DC lines separately. It is verified by both theory and simulation that the proposed method can obtain better planning schemes.

\section{Acknowledgments}

This work was supported by the Science and Technology Project of State Grid Corporation of China (521532190003).

\section{References}

1. Hong C, Xu S K, Zhang, Zhang J S, et al. (2016) Research and application of multiple STATCOMs to improve the stability of AC/DC power systems in China Southern Grid. IET Generation Transmission \& Distribution, 13(10): 3111-3118.

2. Wang F, Li H, Yang L, et al. (2018) Capacity planning method for flexible DC power grid considering access of large-scale new energy. Proceedings of the CSU-EPSA, 30(12): 53-59.

3. Ahmed H M.A.A, Eltantawy A B, Salama M M.A. (2018) A planning approach for the Network Configuration of AC-DC Hybrid Distribution Systems. IEEE Transactions on Smart Grid, 9(3): 2203-2213.

4. Wu Z, Sun Q R, Gu W, et al. (2020) AC/DC hybrid distribution system expansion planning under long-term uncertainty considering flexible investment. IEEE Access, 8: 94956-94967.

5. Zhu J B, Zhou X Y, Zeng P L, et al. (2020) Great Britain's transmission grid planning method and its enlightenment to China's grid planning. Journal of Global Energy Interconnection, 3(1): 59-69.
6. Zhai Z N, Lu Y H, Wan J, et al. (2018) Matchpruning pattern backtracking algorithm for exlcusion constrained workflow satisfiability decision. China Mechanical Engineering, 29(24): 2988-2998.

7. Crampton J, Gutin G, Yeo A. (2013) On the parameterized complexity and kernelization of the workflow satisfiability problem. ACM Transactions on Information and System Security, 16(1): A.1-A.31.

8. Cohen D, Grampton J, Gagarin A, et al. (2014) Iterative plan construction for the workflow satisfiability problem. Journal of Artificial Intelligence Research, 555-577.

9. Daniel K, Andrei G, Gregory G. (2015) Pattern backtracking algorithm for the workflow satisfiability problem. Computer Science, 3(04): 377-381.

10. Wang X F. (1990) Power systems optimization planning. Water Resources and Electric Power Press, Beijing. 